\title{
The Informatics Needs of Amateur Endurance Athletic Coaches
}

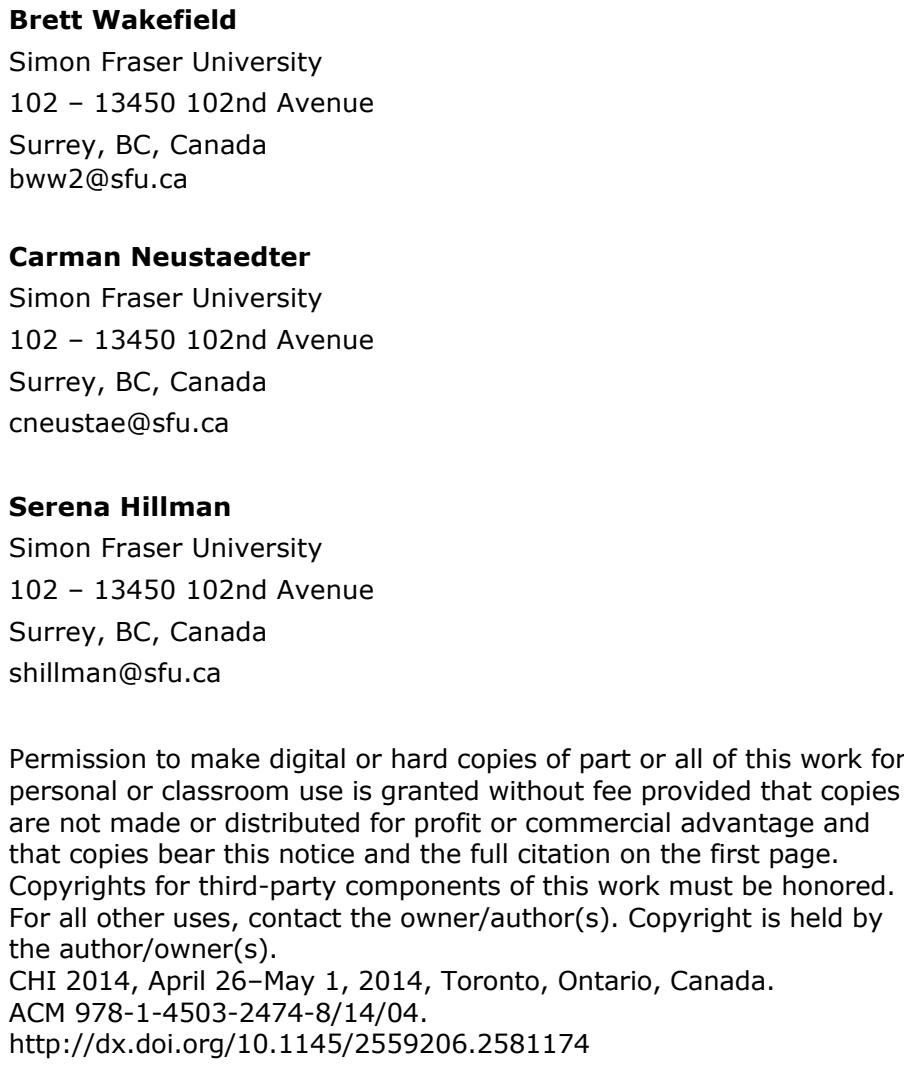

Permission to make digital or hard copies of part or all of this work for personal or classroom use is granted without fee provided that copies are not made or distributed for profit or commercial advantage and that copies bear this notice and the full citation on the first page. Copyrights for third-party components of this work must be honored. For all other uses, contact the owner/author(s). Copyright is held by the author/owner(s).

CHI 2014, April 26-May 1, 2014, Toronto, Ontario, Canada.

ACM 978-1-4503-2474-8/14/04

http://dx.doi.org/10.1145/2559206.2581174

\begin{abstract}
Personal informatics applications are increasingly available for amateur endurance athletes to record and monitor their performance and training. This information can be valuable for coaches who tailor training programs based on this data. Despite this, it is not clear if the information provided by such tools map to the real needs of the amateur athletic community. To address this, we conducted interviews with eight amateur athletic coaches of endurance athletes. Our results show that athlete-specific contextual factors can be important to track and monitor in relation to performance-based metrics. This information can be difficult to capture, analyze, and share. This suggests design opportunities for personal informatics applications for amateur athletes and coaches.
\end{abstract}

\section{Author Keywords}

Athletes; sports; physical activity; personal informatics

\section{ACM Classification Keywords}

H.5.m. Information interfaces and presentation (e.g. HCI): Miscellaneous.

\section{Introduction}

Recent advancements in technology have led to an increase in the adoption of personal informatics systems [4]. One area this has occurred in is the 
endurance athletic community by amateur athletesathletes that train and compete at the amateur level (primarily without pay) who are coached by an experienced person or are self-coached (e.g., high school athletes, college or varsity athletes). Endurance athletics refers to sports that require an athlete to perform over an extended distance or time period [7].

In this area, we see that amateur swimmers, cyclists, and runners often use devices that couple Global Positioning Systems (GPS) with other technologies such as heart rate monitors, power meters, accelerometers, and cadence sensors. As the number of amateur athletes using these smart technologies increases, the opportunity for coaches to analyse or monitor an athlete's performance and adjust the athlete's training program increases accordingly. Thus, even though personal informatics systems are designed for individuals, the data from them may be relevant for other people, such as athletic coaches.

The goal of our research was to learn what types of athlete-related information endurance athletic coaches kept a record of, what record-keeping systems they used (if any), how athletes informed them of trainingrelevant information, and how these records were used to improve an athlete's training. To address this, we conducted in-depth interviews with eight amateur athletic coaches from either a high school or university athletic program. Our results show that while performance metrics are important, additional athletespecific contextual information is valuable for coaches to know about. Yet this information is often challenging to record, share, and analyze. This suggests design opportunities for personal informatics applications for amateur athletes and coaches.

\section{Related Work}

Training is vital to endurance athletics and most athletes train their cardiovascular system multiple times per week to increase performance abilities. Performance levels depend on three main factors: maximal aerobic power (roughly defined as the body's ability to use oxygen), lactate threshold (the point at which lactic acid accumulates in the blood stream and needs to be removed), and economy (the amount of energy expended to produce a particular speed) [6]. Training tries to increase all three of these. A typical goal for endurance athletic training programs is to find a level of workout that pushes the athlete to a maximal performance state without inducing overtraining [2][7]. Training is performed by first overloading the body and pushing it to do more than it normally would. These periods of overloading are followed by a recovery period where an athlete rests herself [7]. Overtime, the body's performance abilities increase because of the overloading [7]. The challenge is figuring out how much the body can handle before overtraining occurs; this can lead to psychological or physiological issues [7].

In order to monitor training, sports medicine literature suggests monitoring five psychological variables: one's general well-being, quality of sleep, success, social stress, and fitness or injury [3]. Coaches can also have athletes assess themselves using the 'Rating of Perceived Exertion' (RPE), originally a numerical scale ranging from 6 (no exertion at all) to 20 (maximal exertion) [1]. We also know that additional factors also affect an athlete's training. This includes one's diet [6][9], water and electrolyte balance, the intake of vitamins and minerals [6], as well as sleep, circadian rhythm, and travel fatigue [9]. Our work builds on this literature to understand what information coaches of 
Table 1. Participant demographics and sport classification.

\begin{tabular}{|l|l|l|l|l|l|}
\hline P & Sex & \multicolumn{1}{|c|}{ Age } & Sport / Experience & \multicolumn{1}{|c|}{ Background } & Coaching \\
\hline 1 & F & $35-45$ & Track \& Cross Country & Former Athlete & Team: 5-6 \\
\hline 2 & F & $55+$ & Track \& Cross Country & Former Elite Level Athlete & Team: 12-24 \\
\hline 3 & M & $35-45$ & Triathalon & Athlete \& Kinesiologist & Team: 5-10 \\
\hline 4 & M & $25-35$ & Track \& Cross Country & Former Athlete & Team: 14-30 \\
\hline 5 & M & $55+$ & Track \& Cross Country & Former Elite Level Athlete & Team: 14-30 \\
\hline 6 & M & $25-35$ & Cycling & Athlete & Individuals: 4 \\
\hline 7 & M & $35-45$ & Cycling & Athlete \& Kinesiologist & Individuals: 6 \\
\hline 8 & M & $35-45$ & Track \& Cross Country & Former Elite Level Athlete & Team: 2 \\
\hline
\end{tabular}

amateur endurance athletes actually find valuable and record, if and how this information is shared between coaches and athletes, and how technology does or does not support this sharing and record keeping.

\section{Study Method}

\section{Participants}

We recruited eight participants (two female) from Vancouver, Canada who had experience coaching amateur endurance athletes. Five participants had coached track and cross-country running teams, two participants had coached individual road cyclists, and one coached a group of triathletes (Table 1 ). While all of the participants tracked some type of athlete-related data for at least a small number of their athletes, only three participants had used athletic web systems and biofeedback devices to do so. Two participants used online systems, such as Google Docs or Training Peaks, to communicate and record workout data, and the rest recorded data with pen and paper, or a combination of paper and Microsoft Excel. This variability was because some participants were less comfortable with technology while others were 'early adopters.'

\section{Interview Method}

We conducted in-depth semi-structured interviews that lasted roughly sixty minutes in length. Interview questions were organized into four different sections: background information, coach-athlete interactions (e.g., "How do you monitor athletes improvements or declines in performance?"), coaching methodology

(e.g., "Do you use any athlete data to structure training programs?"), and technology and personal informatics systems (e.g., "Have you used any personal informatics athletic applications?"). We also showed them a sample workout from Garmin Connect, Strava, and Nike+. We asked them to imagine they were the athlete's coach and analyze the given workouts. We followed this with questions about the value of the data in the applications and its visualization.

Data Analysis

All of the interviews were recorded through handwritten notes and analyzed through thematic analysis. We highlighted and coded recurring themes and then reviewed and iterated on these until we established what we thought were the main findings. Through this analysis, we identified several main results themes.

\section{Performance and Contextual Data}

The most commonly collected data amongst coaches was athletes' distances and time for intervals in a workout, and totals of weekly activity. This provided them with a basis for analysis and comparison of an athlete's performances and improvements. The coaches in our study also identified several areas of athlete-specific contextual information that they felt was important to know and understand in order to evaluate, adapt, and improve an endurance athlete's training. This data was coupled with performance data. 


\begin{tabular}{|c|c|c|c|c|}
\hline Date & Workout & Interval Time & $\begin{array}{c}\text { Weekly } \\
\text { Mileage }\end{array}$ & Comments \\
\hline Sat. 2, 2011 & $800,4 \times 800$ & $2.19 .08,2.23$ & & Sick :( had bad cold- sore throat and bad cough in lungs \\
\hline Sun. 3, 2011 & & & & \\
\hline Tues. 5, 2011 & $2 \times 8004 \times 400 \quad 3^{\prime} 6^{\prime}$ & $\begin{array}{c}2: 42,2: 45,75 \\
76\end{array}$ & & $\begin{array}{l}\text { Still sick- lungs were really tight during worko } \\
\text { couldnt breathe without coughing }\end{array}$ \\
\hline Tues. 12, 2011 & $2 \times 4004 \times 200 \quad 3^{\prime} 6^{\prime}$ & & & $\begin{array}{l}\text { Have final exam @ 3:30, so will not be able to make } \\
\text { practice (will come talk to you on Monday, Marek) }\end{array}$ \\
\hline
\end{tabular}

Figure 1. A portion of an athlete and coach's shared records in Google Docs.

Rate of Perceived Exertion

Participants told us that one of the most common factors that they evaluated was an athlete's RPE. This contextual factor was used in comparison with workout data, such as distance and time, to track an athlete's performance over time. For instance, P8 described that he could use RPE to predict when an athlete was becoming 'overtrained.' Though RPE could help coaches further analyze workout data, only two of the eight participants explicitly recorded this data. Four other participants explained they would verbally communicate with an athlete after the workout was completed to determine RPE or understand how the athlete felt during the training. These coaches chose not to record RPE as they either had a small number of athletes, and felt they could remember such reactions, or had too many athletes and felt the extra transcription was too tedious.

Injuries \& IIlnesses

Seven of eight coaches found it important to record signs of athlete injury or illness. This information was typically communicated by the athlete to the coach with a verbal description and rating of pain on a severity scale. Figure 1 shows the documentation of sickness (in the "Comments" column) by one athlete in a Google Doc file that was shared with her coach. Participants explained how it was important to understand what the injury or illness was to determine how long the athlete would need to rest. This often involved further communication with sports doctors and physiotherapists to diagnose the problem.

The challenge with injuries and illnesses relates to communication. Athletes may not understand the severity of their situation, or know what is important to tell their coach. One participant commented:

"The problem is that they let the injury get bad before they tell me about it, or they don't tell me at all and I find out while at practice... if they communicate these injuries...I can alter their practice..." - P2

A similar issue occurred when there was insufficient communication between the coach, athlete and other stakeholders such as sports doctors or psychologists. Coaches may try to push athletes too hard when they return from an injury, or athletes will fear that they are losing fitness and come back to practice before they are fully healed. Our participants told us that better communication between coaches and sport doctors would help ensure that an athlete's recovery was carried out correctly.

Sleep \& Stress

Our participants told us that sleep and stress were factors that had a tendency to affect an athlete's performance on a specific day of training, rather than a longer period of time. Similarly to injuries and illnesses, the affected athlete usually communicated this information to the coach. Participants explained that athletes that were overtired or 'stressed' risked negative shifts in their mood and mental state. In these situations, coaches would often alter the athlete's workout to be easier or more enjoyable. 
"I altered workouts without the athlete knowing... I evaluate how the athlete is feeling, and change the workout to something that they would enjoy and be able to complete with a positive attitude." - P1

Though all participants mentioned that they evaluated an athlete's sleep or stress, only three of the coaches actually kept record of these factors. Other coaches explained that, while this information was important, it was often tedious to record for every athlete, and provided yet another layer of information to transcribe.

\section{Mood}

An athlete's mood can also have a direct effect on his or her performance. Our participants tried to gauge mood through communication with their athletes. For example, P1, who trained six athletes, explained that she was able to gauge an athlete's mood through subtle conversation before the workout, even if the conversation was unrelated to the training. Because she knew her athletes at a personal level, she could 'read' many of their emotions and predict how they would react to certain workouts. In contrast, P4 explained that with a large team of 20-50 athletes, he used Google Docs to read training notes written by his athletes. From these notes, he could begin to evaluate the effect that the training had on a particular athlete's mood. However, going through notes was very time consuming and it was difficult to know what to look for to determine mood.

\section{Goals and Timelines}

Participants told us that goals and key races, such as a national championship, were also important to track as part of training. Goals and races act as focal points for a competitive season and the primary reason for committing to a training program. The challenge is that goals were often lost track of during a long training period. For example, P3 described a circumstance where his athlete's primary goal or peak race was four years away when the athlete would be qualifying for the Olympic Games. He explained that, psychologically, it was hard to keep an athlete focused when the development towards a goal was long-term. Four coaches told us that giving athletes the ability to visualize their goals in relation to their current training would allow them to see the big picture and mentally adapt to future workouts.

\section{Analysis of Existing Systems}

We also asked participants about existing personal informatics applications and reactions to them were mixed. On one hand, some coaches valued any new opportunities for better record keeping. Yet others felt their existing process was 'good enough.' Participants commented that the applications' ability to record contextual factors was limited as it was most often in the form of textual notes. This was considered difficult to read through and analyze, especially for coaches of large teams. Coaches also found that a design emphasis was placed on individual workouts as opposed to longer term workouts and training that could build up to peak races. They also wanted to see better comparisons between yearly, monthly, and weekly data that could be easily coupled with the contextual factors that had a strong influence on performance metrics.

\section{Discussion and Conclusions}

Our study highlights design opportunities for personal informatics systems for amateur athletic coaching.

First, we see the need to strongly focus designs around the coupling of athlete-specific contextual information 
with performance data. Naturally, this needs to be easy to input and easy to analyze. On one hand, it may be easier for an athlete to record qualitative, textual data (as evidenced by the manner in which they do it currently), yet our study shows this can be difficult for coaches to read, parse, and understand. Coaches of larger teams might miss important data such as injury or fatigue. This suggests design opportunities for providing athletes with an understanding of what information they should document and how to best present it. It also suggests tools that can help coaches easily assess both contextual and performance data. We also see the need to focus on long-term goals and comparisons of performance data to help coaches plan the progression of a training program.

Second, we see design opportunities for information sharing between athletes and coaches. Many coaches relied on face-to-face interactions to gather data. Yet some of the relevant information may come at times when the coach is not around. Athletes must be able to remember this information and convey it to the coach. Some athletes used journals or notes to circumvent this where they would then share the data with the coach at a later point. However, this relies on athletes remembering to record information between workouts and know what is relevant to record. It also means that athletes must remember to present this information to their coaches. Clearly this suggests opportunities for applications that can facilitate information sharing between coaches and athletes inthe-moment or on a more frequent basis. Turning to commercial systems, we see that this is not a current emphasis. Athletes are able to post performance data online for others to see, however, there is no emphasis on athlete-to-coach exchange of information and discussion of contextual information.

\section{References}

[1] Borg, G. Perceived exertion as an indicator of somatic stress, Scandinavian Journal of Rehabilitation Medicine, 2 (2), 92-98, (1970).

[2] Houmard, J.A. Endurance Athletes: What is the Optimal Training Strategy?, International Journal of Sports Medicine, 30(5), Stuttgart (2009).

[3] Kellmann, M. Underrecovery and overtraining: Different concepts, similar impact?, In M. Kellmann (Ed.), Enhancing recovery: Preventing underperformance in athletes, Human Kinetics (2002).

[4] Li, I., Dey, A. K., and Forlizzi, J., Understanding My Data, Myself: Supporting Self-Reflection with Ubicomp Technologies, Proc. Ubicomp, Springer (2011).

[5] Malkinson, T., Current and Emerging technologies in endurance athletic training and race monitoring, Science and Technology for Humanity, IEEE (2009).

[6] Pate, R. and Branch, D. Training for Endurance Sport, Medicine and science in sports and exercise, 24, (1992).

[7] Rearick, M., Creasy, J., and Buriak, J. Avoid Overtraining in Young Athletes, Journal of Physical Education, Recreation \& Dance, 82(5), Academic Alliance for Health, Physical Education, Recreation and Dance, (2011).

[8] Saupe, D., Luchtenberg, D., Roder, M., Federolf, C. Analysis and visualization of space-time variant parameters in endurance sport training, Proc. IACSS, (2007).

[9] Venter, R. Rest, and Specifically Sleep, Play a Major Role in Athletic Performance, South African Journal of Continuing Professional Development, 26(1), (2008). 